\title{
Disyunción maxilar rápida asistida con microtornillos en pacientes en crecimiento con deficiencia maxilar transversal.
}

\section{Miniscrew assisted rapid maxillary expansion in growing patients with transverse maxillary deficiency.}

\author{
Iván Silva-Ruz ${ }^{1,2}$, Fernanda Tort-Barahona ${ }^{1,2}$, Paula Acuña-Aracena ${ }^{1,2}$, Pamela Villalon-Pooley ${ }^{1,2 *}$
}

1. Escuela de Odontología, Facultad de Medicina, Pontificia Universidad Católica de Chile, Santiago, Chile.

2. Proyecto Epistemonikos, Santiago, Chile.

* Correspondencia Autor: Pamela Villalon-Pooley | Dirección: Centro Evidencia UC, Pontificia Universidad Católica de Chile, Diagonal Paraguay 476, Santiago, Chile | E-mail: pvillalon@uc.cl

\section{RESUMEN}

Introducción: La disyunción maxilar rápida tradicional (RME) ha sido el tratamiento de elección para corregir la maloclusión secundaria a la deficiencia maxilar transversal. Debido a las complicaciones que pueden presentar los pacientes, se han desarrollado técnicas de disyunción maxilar asistida por microtornillos (MARPE). Sin embargo, aún no hay consenso respecto a los reales beneficios de la disyunción maxilar rápida asistida por microtornillos por sobre la disyunción tradicional en pacientes jóvenes y en crecimiento. Métodos: Realizamos una búsqueda en Epistemonikos, la mayor base de datos de revisiones sistemáticas en salud, la cual es mantenida mediante el cribado de múltiples fuentes de información, incluyendo MEDLINE, EMBASE, Cochrane, entre otras. Extrajimos los datos desde las revisiones identificadas, analizamos los datos de los estudios primarios, realizamos un metanálisis y preparamos una tabla de resumen de los resultados utilizando el método GRADE. Resultados y conclusiones: Identificamos siete revisiones sistemáticas que en conjunto incluyeron seis estudios primarios, de los cuales todos corresponden a ensayos aleatorizados. Concluimos que: la disyunción maxilar rápida asistida por microtornillos (MARPE) probablemente disminuye la pérdida de hueso alveolar bucal y la resistencia de la vía aérea nasal en comparación a la disyunción maxilar rápida tradicional (RME) (certeza evidencia moderada). MARPE podría disminuir la inclinación dentaria y resultar en poca o nula diferencia en la percepción de dolor en comparación con RME (certeza de la evidencia baja). No es posible establecer con claridad si MARPE aumenta la expansión maxilar en comparación con RME, debido a que la certeza de la evidencia ha sido evaluada como muy baja.

PALABRAS CLAVE

Ortodoncia; Expansion maxilar; Epistemonikos; MARPE; RME; GRADE.

\section{ABSTRACT}

Introduction: Traditional rapid maxillary expansion (RME) has been the gold-standard treatment to correct malocclusion secondary to transverse maxillary deficiency. Due to related complications, mini screw assisted maxillary expansion (MARPE) techniques have been developed. However, there is no consensus regarding the benefits of mini screw assisted maxillary expansion over traditional expansion in young and growing patients. Methods: We searched in Epistemonikos, the largest database of systematic reviews in health, which is maintained by screening multiple information sources, including MEDLINE, EMBASE, Cochrane, among others. We extracted data from the systematic reviews, reanalyzed data of primary studies, conducted a metaanalysis and generated a summary of findings table using the GRADE approach. Results and conclusions: We identified seven systematic reviews including six studies overall, all of which were randomized trials. We concluded that mini screw maxillary expansion (MARPE) probably reduces buccal alveolar bone and nasal airway resistance compared to traditional rapid maxillary expansion (RME). MARPE may reduce dental inclination and result in little or no difference in pain perception compared to RME. We are uncertain whether MARPE improves maxillary expansion compared to RME, as the certainty of the evidence has been assessed as very low.

\section{KEY WORDS}

Orthodontics; Maxillary expansion; Epistemonikos; MARPE; RME; GRADE.

Int. J. Inter. Dent Vol. 14(1); 61-66, 2021. 


\section{PROBLEMA}

Uno de los tratamientos de ortodoncia para la corrección de la mordida cruzada (uni/bilateral) secundaria a la deficiencia maxilar transversal, es la disyunción maxilar rápida, ya que logra estimular el crecimiento del maxilar al transmitir grandes fuerzas hacia la red de suturas circunmaxilares ${ }^{(1,3,4,5,6,7)}$. Para que esto ocurra, es gravitante que las suturas aún sean susceptibles (estén abiertas, permeables, poco imbricadas) a los estímulos mecánicos que transmiten los aparatos de ortodoncia, condición particularmente esperable en pacientes jóvenes y en crecimiento ${ }^{(4,6)}$.

Para lograr la disyunción, tradicionalmente se utilizan aparatos con anclaje a los dientes o a los dientes y al tejido blando. Ambos son capaces de producir una separación de la sutura media palatina, resultando en una expansión esqueletal del maxilar. Sin embargo, estas fuerzas pueden producir eventos adversos como aumentos en la inclinación dental, reabsorción de raíces, reabsorción del hueso alveolar y alteraciones en los tejidos periodontales ${ }^{(1,2,3,4,5)}$. Para intentar un mejor manejo de estas complicaciones, se han diseñado nuevos aparatos que se anclan directamente a la superficie de los huesos -o- a los huesos y dientes (híbridos) mediante microtornillos, permitiendo una transmisión de la fuerza con una mejor distribución sobre la superficie palatina, y por lo tanto, con efectos más directos en las suturas ${ }^{(1,2,4,5)}$. Esto permitiría lograr una disyunción maxilar efectiva, con menor inclinación dentaria y menor stress en los tejidos periodontales ${ }^{(3,4,5)}$.

No obstante, la evidencia respecto al uso de la disyunción maxilar rápida asistida por microtornillos (MARPE) por sobre la disyunción maxilar rápida tradicional (RME) es controversial y aún existe incertidumbre acerca de la seguridad y efectividad que podría tener el uso de aparatos con anclaje óseo ${ }^{(1,2,3,4,5)}$.

\section{MÉTODOS}

Realizamos una búsqueda en Epistemonikos, la mayor base de datos de revisiones sistemáticas en salud, la cual es mantenida mediante búsquedas en múltiples fuentes de información, incluyendo MEDLINE, EMBASE, Cochrane, entre otras. Extrajimos los datos desde las revisiones identificadas y analizamos los datos de los estudios primarios. Con esta información, generamos un resumen estructurado denominado FRISBEE (Friendly Summaries of Body of Evidence using Epistemonikos), siguiendo un formato preestablecido, que incluye mensajes clave, un resumen del conjunto de evidencia (presentado como matriz de evidencia en Epistemonikos), metanálisis del total de los estudios cuando sea posible, una tabla de resumen de resultados con el método GRADE y una sección de otras consideraciones para la toma de decisión.

\section{Mensajes clave}

- La disyunción maxilar rápida asistida por microtornillos (MARPE) probablemente disminuye la pérdida de hueso alveolar bucal y la resistencia de la vía aérea nasal en comparación a la disyunción maxilar rápida tradicional (RME)

- MARPE podría disminuir la inclinación dentaria y podría resultar en poca o nula diferencia en la percepción de dolor en comparación con RME (certeza de la evidencia baja).

- No es posible establecer con claridad si MARPE aumenta la expansión maxilar en comparación con RME, debido a que la certeza de la evidencia ha sido evaluada como muy baja.

Acerca del conjunto de evidencia para esta pregunta

\begin{tabular}{|c|c|}
\hline Cuál es la evidencia & $\begin{array}{l}\text { Encontramos siete revisiones } \\
\text { sistemáticas }(1,2,3,4,5,6,7) \text {, que incluyeron }\end{array}$ \\
\hline $\begin{array}{l}\text { Véase matriz de evidencia } \\
\text { en Epistemonikos más } \\
\text { abajo. }\end{array}$ & $\begin{array}{l}\text { seis estudios primarios reportados } \\
\text { en } 11 \text { referencias }(8,9,10,11,12,13,14,15,16,17 \\
\text { 18) de los cuales todos son ensayos } \\
\text { aleatorizados. }\end{array}$ \\
\hline
\end{tabular}

Todos los ensayos incluyeron a pacientes con deficiencia maxilar transversal que requerían disyunción maxilar. La edad de los y las participantes varió entre los 8 y 17 años. El porcentaje de mujeres varió desde un $52 \%$ a un $69,4 \%$ entre los ensayos $(8,9,11,12,14,15)$.

Qué tipo de pacientes incluyeron los estudios*

Dentro de los criterios de exclusión, 5 ensayos $(8,9,11,14,15)$ excluyeron a pacientes con características sindrómicas o enfermedades sistémicas y 5 ensayos ${ }^{(9,11,12,14,15)}$ excluyeron a pacientes con tratamiento de ortodoncia previo.

Todos los ensayos $(8,9,11,12,14,15)$ compararon disyunción maxilar rápida asistida con microtornillos (MARPE) con disyunción maxilar rápida tradicional (RME).
Qué tipo de intervenciones incluyeron los estudios*
Los ensayos ${ }^{(8,9)} y^{(11)}$ describieron los diseños de los aparatos para realizar RME y MARPE.

En todos los ensayos RME fue realizada con un disyuntor tipo Hyrax (anclaje a los dientes), mientras que MARPE fue soportada por dos microtornillos, excepto en un ensayo (11) que fue soportada por 4 microtornillos.

Los ensayos reportaron múltiples desenlaces, los cuales fueron agrupados por las revisiones sistemáticas de la siguiente manera:

- Flujo aéreo nasal, medido con rinomanometría $\left(\mathrm{cm}^{3} / \mathrm{s}\right)$

- Resistencia de la vía aérea nasal, medido con rinomanometría $\left(\mathrm{Pa} \mathrm{s} / \mathrm{cm}^{3}\right)$

- Expansión maxilar, medido a través de cone beam $(\mathrm{mm})$

Qué tipo de desenlaces - Inclinación dental premolar, medido a través de cone beam (grados)

- Dolor, medido en escala visual análoga 1-100 (mm)

- Pérdida del hueso alveolar bucal premolar, medido a través de cone beam $(\mathrm{mm})$.

El seguimiento promedio de los ensayos (posterior a la expansión) fue de 6 meses $(8,9,11,12,14)$ con un rango que fluctuó entre los 3 y 18 meses. Un ensayo no especificó el seguimiento (15).

* La información sobre los estudios primarios es extraída desde las revisiones sistemáticas identificadas, no directamente desde los estudios, a menos que se especifique lo contrario.

\section{RESUMEN DE LOS RESULTADOS}

La información sobre los efectos de la disyunción maxilar rápida asistida por microtornillos (MARPE) comparada con la disyunción maxilar rápida tradicional (RME) en pacientes jóvenes y en crecimiento con deficiencia maxilar transversal está basada en seis ensayos $(9,11,12,14,15,16)$ que incluyeron a 268 pacientes.

Tres ensayos evaluaron la expansión maxilar (106 pacientes) $)^{(8,9,11)}$, dos ensayos reportaron la pérdida del hueso alveolar premolar $(65$ participantes) ${ }^{(9,11)}$, un ensayo evaluó la resistencia y flujo de la vía aérea nasal (30 pacientes) ${ }^{(15)}$, un ensayo midió el dolor asociado al tratamiento (41 pacientes) ${ }^{(8)}$ y un ensayo midió la inclinación dental premolar (41 pacientes) ${ }^{(13)}$. 
El resumen de los resultados es el siguiente:

- MARPE probablemente disminuye la resistencia de las vía aérea nasal en comparación con RME.

- No es posible establecer con claridad si MARPE aumenta la expansión maxilar en comparación con RME, debido a que la certeza de la evidencia ha sido evaluada como muy baja.
- MARPE podría disminuir la inclinación dentaria en comparación con RME (la certeza de la evidencia es baja).

- MARPE podría resultar en poca o nula diferencia en la percepción de dolor en comparación con RME (certeza de la evidencia baja).

- MARPE probablemente disminuye la pérdida de espesor del hueso alveolar bucal en comparación con el RME.

\begin{tabular}{|c|c|c|c|}
\hline \multicolumn{4}{|c|}{$\begin{array}{l}\text { Disyunción maxilar rápida en pacientes jóvenes que presentan deficiencia } \\
\text { maxilar transversal }\end{array}$} \\
\hline $\begin{array}{l}\text { Pacientes } \\
\text { Intervención } \\
\text { Comparación }\end{array}$ & \multicolumn{3}{|c|}{$\begin{array}{l}\text { Pacientes jóvenes y en crecimiento que presentan deficiencia maxilar } \\
\text { transversal } \\
\text { Disyunción maxilar rápida asistida con microtornillos (MARPE) } \\
\text { Disyunción maxilar rápida tradicional (RME) }\end{array}$} \\
\hline \multirow[b]{2}{*}{ Desenlaces } & \multicolumn{2}{|c|}{ Efecto absoluto* } & \multirow{2}{*}{$\begin{array}{l}\text { Certeza de la evidencia } \\
\text { (GRADE) }\end{array}$} \\
\hline & $\begin{array}{l}\text { CON } \\
\text { RME }\end{array}$ & $\begin{array}{l}\text { CON } \\
\text { MARPE }\end{array}$ & \\
\hline \multirow{2}{*}{$\begin{array}{l}\text { Resistencia vía } \\
\text { aérea nasal ( } \mathrm{Pa} \\
\left.\mathrm{s} / \mathrm{cm}^{3}\right)^{* *}\end{array}$} & $0,89 \mathrm{~Pa} \mathrm{~s} / \mathrm{cm} 3$ & $0,66 \mathrm{~Pa} \mathrm{~s} / \mathrm{cm} 3$ & \multirow{2}{*}{$\begin{array}{c}\oplus \oplus \oplus \bigcirc \\
\text { Moderada }^{1}\end{array}$} \\
\hline & \multicolumn{2}{|c|}{$\begin{array}{c}\text { DM: } 0,23 \text { menos } \\
\text { (Margen de error: } 0,04 \text { a } 0,42 \text { menos) }\end{array}$} & \\
\hline \multirow{2}{*}{$\begin{array}{l}\text { Expansión } \\
\text { maxilar*** }\end{array}$} & \multicolumn{2}{|c|}{$\begin{array}{l}\text { La escala de expansión maxilar fue en } \\
\text { promedio } 0,7 \text { desviaciones estándar mayor } \\
\text { en el grupo con MARPE }\end{array}$} & \multirow{2}{*}{$\begin{array}{l}\oplus \bigcirc \bigcirc \\
\text { Muy baja }\end{array}$} \\
\hline & \multicolumn{2}{|c|}{$\begin{array}{c}\text { DME: } 0,7 \\
\text { (Margen de error: } 0,59 \text { menos a } 2,0 \text { más) }\end{array}$} & \\
\hline \multirow{2}{*}{$\begin{array}{l}\text { Inclinación } \\
\text { dental } \\
\text { premolar } \\
\text { (grados)**** }\end{array}$} & $-0,64$ grados & $-5,7$ grados & \multirow[b]{2}{*}{$\underset{\text { Baja }^{1,2}}{\oplus \oplus \bigcirc \bigcirc}$} \\
\hline & \multicolumn{2}{|c|}{$\begin{array}{l}\text { DM: 5,06 grados menos } \\
\text { (Margen de error: 6,93 menos a 3,19 más) }\end{array}$} & \\
\hline \multirow{2}{*}{$\begin{array}{l}\text { Dolor } \\
(\mathrm{mm}) * * * * *\end{array}$} & $19,7 \mathrm{~mm}$ & $24 \mathrm{~mm}$ & \multirow{2}{*}{$\begin{array}{l}\oplus \oplus \bigcirc \bigcirc \\
\text { Baja }^{1,2}\end{array}$} \\
\hline & (Margen de er & $\begin{array}{l}3 \text { mm más } \\
, 66 \text { a 6,94 más) }\end{array}$ & \\
\hline \multirow{2}{*}{$\begin{array}{l}\text { Pérdida del } \\
\text { hueso alveolar } \\
\text { bucal } \\
\text { premolar*** }\end{array}$} & $\begin{array}{l}\text { La escala de pé } \\
\text { bucal fue en pro } \\
\text { estándar menor }\end{array}$ & $\begin{array}{l}\text { de hueso alveolar } \\
0,75 \text { desviaciones } \\
\text { grupo con MARPE }\end{array}$ & \multirow{2}{*}{$\begin{array}{c}\oplus \oplus \oplus \bigcirc \\
\text { Moderada }^{1}\end{array}$} \\
\hline & \multicolumn{2}{|c|}{$\begin{array}{c}\text { DME: } 0,75 \text { menos } \\
\text { (Margen de error: } 0,24 \text { a } 1,25 \text { menos) }\end{array}$} & \\
\hline
\end{tabular}


Margen de error: Intervalo de confianza del 95\% (IC 95\%).

DM: Diferencia de medias.

DME: Diferencia de medias estandarizada.

GRADE: Grados de evidencia del GRADE Working Group (ver más adelante).

*El promedio con RME está basado en el promedio del estudio de mayor peso. El promedio con MARPE (y su margen de error) está calculado a partir de la diferencia de medias (y su margen de error).

** La resistencia de la vía aérea nasal se midió con rinomanometría y su unidad de medida es Pa $\mathrm{s} / \mathrm{cm}^{3}$. A menor $\mathrm{Pa} \mathrm{s} / \mathrm{cm}^{3}$, es menor la resistencia de la vía aérea nasal. Una reducción en la resistencia de $0,21 \mathrm{~Pa} \mathrm{~s} / \mathrm{cm}^{3}$ se consideró como un efecto clínicamente relevante [15].

$* * *$ La diferencia media estandarizada se utiliza cuando el desenlace ha sido medido en diferentes escalas, siendo difícil su interpretación clínica. Comúnmente se acepta que valores cercanos a 0,2 tendrían poca relevancia clínica, valores de 0,5 tendrían relevancia moderada (se reconoce clínicamente) y valores superiores a 0,8 tendrían relevancia alta.

$* * * *$ Medido en los premolares superiores izquierdos. La unidad de medida es grados. A menos grados, menor la inclinación dentaria.

*****Medido en escala visual análoga de 1 a $100 \mathrm{~mm}$. Se considera que a mayor puntaje, más dolor.

${ }^{1}$ Se disminuyó un nivel de certeza de evidencia por imprecisión, ya que debido al número de estudios y tamaño muestral, no se puede descartar que el efecto observado sea producto del azar. En el caso del desenlace "Expansión maxilar, se decidió disminuir por imprecisión, ya que a cada extremo del intervalo de confianza se tomarían decisiones diferentes.

${ }^{2}$ Se disminuyó un nivel de certeza de evidencia por riesgo de sesgo, ya que se observaron limitaciones en el ciego, en la generación de la secuencia de aleatorización y/o en la descripción de los abandonos.

${ }^{3}$ Se disminuyó un nivel de certeza de evidencia por inconsistencia, ya que los ensayos presentan conclusiones diferentes $\left(\mathrm{I}^{2}=90 \%\right)$.

Siga el enlace para acceder a la versión interactiva de esta tabla (Interactive Summary of Findings - iSoF)

\section{Acerca de la certeza de la evidencia (GRADE)*}

\section{$\oplus \oplus \oplus \oplus$}

Alta: La investigación entrega una muy buena indicación del efecto probable. La probabilidad de que el efecto sea sustancialmente distinto ${ }^{+}$es baja.

$\oplus \oplus \oplus \bigcirc$

Moderada: La investigación entrega una buena indicación del efecto probable. La probabilidad de que el efecto sea sustancialmente distinto† es moderada.

\section{$\oplus \oplus \bigcirc$}

Baja: La investigación entrega alguna indicación del efecto probable. Sin embargo, la probabilidad de que el efecto sea sustancialmente distinto' es alta.

\section{$\oplus 000$}

Muy baja: La investigación no entrega una estimación confiable del efecto probable. La probabilidad de que el efecto sea sustancialmente distinto† es muy alta.

*Esto es también denominado 'calidad de la evidencia' o 'confianza en los estimadores del efecto'. +Sustancialmente distinto = una diferencia suficientemente grande como para afectar la decisión 


\section{OTRAS CONSIDERACIONES PARA LA TOMA DE DECISIÓN}

\section{A quién se aplica y a quién no se aplica esta evidencia}

- Estos resultados aplican para pacientes jóvenes y/o en crecimiento ( 9 a 17 años), sin distinción de sexo, sin tratamientos de ortodoncia previos, sanos y que presenten deficiencia maxilar en sentido transversal.

- Estos resultados no aplican para pacientes que necesitan una disyunción maxilar rápida asistida quirúrgicamente (SARME).

\section{Sobre los desenlaces incluidos en este resumen}

- La mayoría de los desenlaces seleccionados son considerados críticos para la toma de decisión de acuerdo a la opinión de los autores de este resumen, coincidiendo en general con los evaluados por las revisiones sistemáticas identificadas.

- Los desenlaces "pérdida de hueso alveolar bucal premolar" e "inclinación dental premolar" son críticos para la toma de decisión ya que ambos son ampliamente reconocidos como eventos adversos de la disyunción maxilar rápida tradicional (RME) y han inspirado que nuevos tratamientos -como la disyunción maxilar rápida asistida por microtornillos (MARPE)- vayan en busca de soluciones que disminuyan estos eventos adversos para los y las pacientes.

- Es necesario establecer desenlaces principales (core outcome set) para ensayos clínicos sobre la disyunción maxilar rápida que sean medidos a través de instrumentos válidos, sensibles y que sean relevantes y perceptibles por los y las pacientes. Además, debe considerarse la inclusión de desenlaces reportados por el/la paciente, como la calidad de vida, función masticatoria, función fonética y dificultad para realizar higiene oral durante el tratamiento.

\section{Balance daño/beneficio y certeza de la evidencia}

- Según la evidencia disponible MARPE probablemente disminuye la pérdida de hueso alveolar bucal y podría disminuir la inclinación dentaria en comparación a RME. Además, MARPE probablemente mejora la permeabilidad de la vía aérea (disminuyendo su resistencia) en comparación a RME, pero la certeza de la evidencia es baja. Por otro lado, no existen diferencias clínicamente importantes en el dolor percibido por los y las pacientes entre MARPE y RME.

- No es posible establecer con claridad si el uso de MARPE aumenta la expansión maxilar, debido a que la certeza de la evidencia sobre la expansión maxilar fue muy baja, por lo que actualmente no se puede demostrar beneficios de una terapia por sobre la otra.

- Finalmente, se podría concluir que la evidencia disponible muestra un posible beneficio de MARPE en comparación con RME. Sin embargo, debido a la incertidumbre existente en relación a ciertos desenlaces la elección final debe ser individualizada para cada caso.

\section{Consideraciones de recursos}

- Ninguno de los estudios incluidos realizó un análisis sobre los costos económicos de MARPE y RME. Por lo tanto, se requieren ensayos que analicen específicamente los costos y beneficios de ambos tratamientos.

- A pesar de lo anterior, hay que considerar que la aplicación de microtornillos en niños, niñas o pacientes con poca capacidad de cooperación, necesitarán que la instalación sea llevada a cabo en pabellón por un cirujano o especialista entrenado, con apoyo de guías quirúrgicas y/o sedación. Todo esto encarece el costo del tratamiento y tiene una alta carga emocional para el/la paciente y su familia.

\section{Qué piensan los pacientes y sus tratantes}

- En general, los tratantes consideran que, independiente del costo más elevado y la necesidad de pabellón, en un paciente que esté finalizando su crecimiento (adolescencia tardía) se justifica la decisión de instalar microtornillos dadas las características que adquieren las suturas durante este proceso (suturas más imbricadas y menos permeables) ${ }^{(4,6)}$. En un paciente en crecimiento de menor edad (alrededor de los 10 años), no se justificaría la instalación de microtornillos para realizar la disyunción con MARPE, ya que RME cubre las necesidades.

- Enfrentados a la evidencia presentada en este resumen, la mayoría de los y las pacientes suelen mostrar preferencia por el disyuntor tradicional (RME), ya que al ser una técnica que no requiere instalación de microtornillos, se torna un tratamiento menos impactante en términos emocionales (al no requerir aplicación de anestesia local, ingresar a pabellón en algunos casos o incluso requerir de sedación) y menos costoso. Sin embargo, en base a la evidencia existente, la toma de decisiones debiese considerar las características biológicas del/de la paciente y en la etapa del crecimiento en la que se encuentra, apoyándose en los exámenes complementarios imagenológicos para contrastar el estadío de maduración de la sutura media palatina.

\section{Diferencias entre este resumen y otras fuentes}

- Las conclusiones de este resumen concuerdan con las siguientes revisiones sistemáticas identificadas ${ }^{(1,2,3,4,5)}$, las cuales sugieren que existen potenciales beneficios al realizar MARPE por sobre RME. Sin embargo, declaran que -debido a las limitaciones metodológicas en los estudios incluidos- las recomendaciones y los resultados deben ser considerados con cautela y la elección final de la técnica es dependiente del operador, las características esqueléticas de los y las pacientes y sus preferencias.

- Las revisiones ${ }^{(6,7)}$ reportaron que no existían diferencias significativas entre MARPE y RME, ya que con ambas intervenciones se lograban resultados similares. Sin embargo, estas revisiones basan sus conclusiones en los resultados de un único ensayo ${ }^{(8)}$.

- El resultado de este resumen en relación al desenlace de dolor es discordante con lo afirmado por una de las revisiones sistemáticas incluídas (1), pues ésta concluyó que MARPE se asoció con menos molestias para el paciente durante los primeros días de activación. Esta discordancia se explica porque el outcome dolor en esta revisión sistemática ${ }^{(1)}$ solo es evaluado a través de un ensayo ${ }^{(14)}$ cuyo artículo no reporta los datos necesarios para poder realizar un metaanálisis. Además, este ensayo (14) midió el dolor únicamente en la primera semana de tratamiento, por el contrario, el ensayo ${ }^{(12)}$ utilizado por este resumen mide la experiencia de dolor percibida en la primera activación, una vez durante la expansión y al final de la expansión, describiendo la experiencia en general.

- No se identificaron guías de práctica clínica que realizaran recomendaciones al respecto.

\section{¿Puede que cambie esta información en el futuro?}

- La certeza de la evidencia respecto a la resistencia flujo aéreo nasal y pérdida de hueso alveolar bucal es moderada, por lo que esta evidencia podría cambiar en el futuro. Por otra parte, la evidencia que respalda que MARPE generaría menor inclinación dental que RME es de certeza baja, por lo cual probablemente esta evidencia cambie en el futuro. Finalmente, la evidencia con respecto al desenlace de la expansión maxilar es muy probable que cambie.

- No identificamos ningún ensayo clínico en curso en la International Clinical Trials Registry Platform de la Organización Mundial de la Salud.

- No identificamos ninguna revisión sistemática en curso en el International Prospective register of systematic reviews (PROSPERO) del National Institute for Health Research.

\section{CÓMO REALIZAMOS ESTE RESUMEN}

Mediante métodos automatizados y colaborativos recopilamos toda la evidencia relevante para la pregunta de interés y la presentamos en una matriz de evidencia.

Siga el enlace para acceder a la versión interactiva:

Disyunción maxilar rápida asistida por microtornillos (MARPE) comparado con disyunción maxilar rápida tradicional (RME) en pacientes jóvenes y en crecimiento con deficiencia maxilar transversal

\section{NOTAS}

Si con posterioridad a la publicación de este resumen se publican nuevas revisiones sistemáticas sobre este tema, en la parte superior de la matriz se mostrará un aviso de "nueva evidencia".

Este artículo es parte del proyecto síntesis de evidencia de Epistemonikos. Se elabora con una metodología preestablecida, siguiendo rigurosos estándares metodológicos y proceso de revisión por pares interno. Cada uno de estos artículos corresponde a un resumen, denominado FRISBEE (Friendly Summary of Body of Evidence using Epistemonikos), cuyo principal objetivo es sintetizar el conjunto de evidencia de una pregunta específica, en un formato amigable a los profesionales clínicos. Sus principales recursos se basan en la matriz de evidencia de Epistemonikos y análisis de resultados usando metodología GRADE. Mayores detalles de los métodos para elaborar este FRISBEE están descritos aquí (http://dx.doi.org/10.5867/medwave.2014.06.5997)

La Fundación Epistemonikos es una organización que busca acercar la información a quienes toman decisiones en salud, mediante el uso de tecnologías. Su principal desarrollo es la base de datos Epistemonikos (www.epistemonikos.org).

\section{DECLARACIÓN DE CONFLICTOS DE INTERESES}

Los autores declaran no tener conflictos de intereses con la materia de este artículo.

\section{AGRADECIMIENTOS}

Este resumen de evidencia fue elaborado con el apoyo metodológico del Centro Evidencia UC, Facultad de Medicina, Pontificia Universidad Católica de Chile. 


\section{Bibliografía}

1. Krüsi M, Eliades T, Papageorgiou SN. Are there benefits from using bone-borne maxillary expansion instead of tooth-borne maxillary expansion? A systematic review with meta-analysis. Prog Orthod. 2019 Feb 25;20(1):9.

2. Khosravi M, Ugolini A, Miresmaeili A, Mirzaei H, Shahidi-Zandi V, Soheilifar S, et al. Tooth-borne versus bone-borne rapid maxillary expansion for transverse maxillary deficiency: A systematic review. Int Orthod. 2019 Sep;17(3):425-36.

3. Mashreghi A, Bardideh E, Shafaee H, Dadgarmoghaddam M. Expansión maxilar transmitida por el hueso y expansión maxilar rápida tradicional: una revisión sistemática y un metaanálisis. Revista de Materiales y Técnicas Dentales, 2019; 8(4):159-68.

4. Copello FM, Marañón-Vásquez GA, Brunetto DP, Caldas LD, Masterson D, Maia LC, et al. Is the buccal alveolar bone less affected by mini-implant assisted rapid palatal expansion than by conventional rapid palatal expansion?-A systematic review and meta-analysis. Orthod Craniofac Res. 2020 Aug;23(3):237-49.

5. Algharbi M, Bazargani F, Dimberg L. Do different maxillary expansion appliances influence the outcomes of the treatment? Eur J Orthod. 2018 Jan 23;40(1):97-106 doi: 10.1093/ejo/cjx035.

6. Agostino P, Ugolini A, Signori A, Silvestrini-Biavati A, Harrison JE, Riley P Orthodontic treatment for posterior crossbites. Cochrane Database Syst Rev. 2014 Aug 8;(8):CD000979.

7. Zuccati G, Casci S, Doldo T, Clauser C. Expansion of maxillary arches with crossbite: a systematic review of RCTs in the last 12 years. Eur J Orthod. 2013 Feb;35(1):29-37.

8. Lagravère MO, Carey J, Heo G, Toogood RW, Major PW. Transverse, vertical, and anteroposterior changes from bone-anchored maxillary expansion vs traditional rapid maxillary expansion: a randomized clinical trial. Am J Orthod Dentofacial Orthop. 2010 Mar;137(3):304.e1-12; discussion 304-5

9. Gunyuz Toklu M, Germec-Cakan D, Tozlu M. Periodontal, dentoalveolar, and skeletal effects of tooth-borne and tooth-bone-borne expansion appliances. Am J
Orthod Dentofac Orthop. 2015;148:97-109.

10. Kabalan O, Gordon J, Heo G, Lagravère MO. Nasal airway changes in boneborne and tooth-borne rapid maxillary expansion treatments. Int Orthod. 2015 Mar;13(1):1-15

11. Celenk-Koca T, Erdinc AE, Hazar S, Harris L, English JD, Akyalcin S. Evaluation of miniscrew-supported rapid maxillary expansion in adolescents: A prospective randomized clinical trial. Angle Orthod. 2018 Nov;88(6):702-9.

12. Canan S, Şenışık NE. Comparison of the treatment effects of different rapid maxillary expansion devices on the maxilla and the mandible. Part 1: Evaluation of dentoalveolar changes. Am J Orthod Dentofacial Orthop. 2017 Jun:151(6):1125-38. 13. Lagravère MO, Gamble J, Major PW, Heo G. Transverse dental changes after tooth-borne and bone-borne maxillary expansion. Int Orthod. 2013 Mar;11(1):21-34 English, French.

14. Feldmann I, Bazargani F. Pain and discomfort during the first week of rapid maxillary expansion (RME) using two different RME appliances: A randomized controlled trial. Angle Orthod. 2017 May;87(3):391-6.

15. Bazargani $F$ Magnuson A Ludwig B. Effects on nasal airflow and resistance using two different RME appliances: a randomized controlled trial. Eur J Orthod. 2018 May 25;40(3):281-4.

16. Lagrevère MOV. Analysis of skeletal and dental changes with a tooth- borne and a bone-borne maxillary expansion appliance assessed through digital volumetric imaging. PhD Thesis. University of Alberta. 2009

17. Stepanko LS, Lagravère MO. Sphenoid bone changes in rapid maxillary expansion assessed with cone-beam computed tomography. Korean J Orthod. 2016 Sep;46(5):269-79.

18. Forst DD. External root resorption associated with maxillary expansion therapies as evaluated via cone beam computed tomography: a retrospective randomized clinical trial, Master thesis. Alberta: University of Alberta; 2015. 